\title{
The Use of Tissue Engineering to Fabricate Perfusable 3D Brain Microvessels in vitro
}

\author{
Kalpani N. Udeni Galpayage Dona', Jonathan Franklin Hale1, Tobi Salako', \\ Akanksha Anandanatarajan', Kiet A. Tran'², Brandon J. DeOre², Peter Adam Galie², \\ Servio Heybert Ramirez ${ }^{1,3,4}$ and Allison Michelle Andrews ${ }^{1,3 *}$
}

\begin{abstract}
'Department of Pathology and Laboratory Medicine, Lewis Katz School of Medicine at Temple University, Philadelphia, PA, United States, ${ }^{2}$ Department of Biomedical Engineering, Rowan University, Glassboro, NJ, United States, ${ }^{3}$ The Center for Substance Abuse Research, Lewis Katz School of Medicine at Temple University, Philadelphia, PA, United States,

${ }^{4}$ Shriners Hospitals Pediatric Research Center, Philadelphia, PA, United States
\end{abstract}

\section{OPEN ACCESS}

Edited by:

Clotilde Lecrux,

McGill University, Canada

Reviewed by:

Dan Predescu,

Rush University, United States

William F. Jackson,

Michigan State University,

United States

${ }^{*}$ Correspondence:

Allison Michelle Andrews

allison.andrews@temple.edu

Specialty section:

This article was submitted to

Vascular Physiology,

a section of the journal

Frontiers in Physiology

Received: 26 May 2021 Accepted: 10 August 2021 Published: 31 August 2021

Citation:

Galpayage Dona KNU, Hale JF, Salako T, Anandanatarajan A,

Tran KA, DeOre BJ, Galie PA,

Ramirez SH and Andrews AM (2021)

The Use of Tissue Engineering

to Fabricate Perfusable 3D Brain

Microvessels in vitro.

Front. Physiol. 12:715431.

doi: 10.3389/fphys.2021.715431
Tissue engineering of the blood-brain barrier (BBB) in vitro has been rapidly expanding to address the challenges of mimicking the native structure and function of the BBB. Most of these models utilize 2D conventional microfluidic techniques. However, 3D microvascular models offer the potential to more closely recapitulate the cytoarchitecture and multicellular arrangement of in vivo microvasculature, and also can recreate branching and network topologies of the vascular bed. In this perspective, we discuss current 3D brain microvessel modeling techniques including templating, printing, and self-assembling capillary networks. Furthermore, we address the use of biological matrices and fluid dynamics. Finally, key challenges are identified along with future directions that will improve development of next generation of brain microvasculature models.

Keywords: BBB, NVU, microfluidics, bioengineering, brain endothelial cells

\section{INTRODUCTION}

The blood-brain barrier (BBB) is a highly organized and dynamic structure that acts as a physiological barrier between the central nervous system (CNS) and cerebral blood flow. It continuously regulates the passage of molecules and ions at the capillary level to maintain brain homeostasis (Abbott, 2013). Specialized brain microvascular endothelial cells (BMVECs) with distinct barrier functionalities distinguish the brain capillaries from other peripheral vessels due to the presence of highly developed and organized tight junctional complexes (TJCs) as well as molecular transporters (Bazzoni and Dejana, 2004; Saili et al., 2017; Castro Dias et al., 2019). The cell-cell interaction of BMVECs influences most molecular traffic to take a transcellular route, from the luminal (apical) to the abluminal (basolateral) side of ECs, which provides selectivity for the barrier (Abbott, 2013; Lochhead et al., 2020). Additionally, the increased contact points of TJs restrict movement in the plasma membrane from the apical and basolateral side, contributing to a polarized expression of proteins (Bazzoni and Dejana, 2004). Entry for molecules, like glucose, pass through GLUT1 transporters while large hydrophilic molecules like peptides and proteins are generally restricted, unless shuttled through receptor-mediated transcytosis (Bickel et al., 2001). BMVECs also express multiple efflux pumps on their luminal surface that regulate uptake of larger 
lipophilic molecules, including many drugs, into the brain (Abbott et al., 2010). Conversely, blood gases like CO2 and $\mathrm{O} 2$, diffuse freely across the lipid membrane down their concentration gradients.

In addition to the BMVECs, neurons, glial cells, pericytes, and the astrocytic end-feet that surround vessels form a functional unit (Abbott et al., 2006). Pericytes embedded in the basement membrane wrap around the endothelium and contribute to astrocytic polarization (Bonkowski et al., 2011; Gundersen et al., 2014). The BBB is not a standalone structure; supporting cells play a significant role in the signaling of $\mathrm{BBB}$ modalities to regulate the cerebral microenvironment under dynamic physiological or pathological conditions. Together, the association of these components form what is known as the “neurovascular unit” (NVU) (Zhang et al., 2012).

For decades, animal and 2D models have advanced our understanding of $\mathrm{BBB}$ biology. While informative, in vivo studies are resource and time consuming, and the species differences between humans and animal have minimized the translatability of the results to clinical applications. A lack of predictive outcomes is attributed in part due to diverging features like cellcell signaling, transporter expression, and other physiological differences (Aday et al., 2016; Song et al., 2020). In vitro models, offer the ability to use human primary cells, which can bridge the gap to clinical translation by minimizing species differences. Additionally, in vitro models are tunable and provide a platform to finely control various aspect of BBB properties. However, planar in vitro models fail to mimic the complex features of the NVU. The most commonly used Boyden chamber system, consisting of a porous membrane separating two compartments, fails to facilitate the cylindrical geometry of in vivo vasculature as well as dynamic mechanical stimuli exerted by fluid flow. Shear stress and cyclic strain caused by physiological fluid flow have been shown to be critical to microvascular function and high trans-endothelial electric resistance (TEER) values (Colgan et al., 2007; Cucullo et al., 2013). Furthermore, many organ-ona-chip platforms are based on 2D models of the BBB that cannot replicate the complex network topology of vessels formed in vivo. Thus, there is a pressing need to develop a functional in vitro model that recapitulates the key relationships between cells and the dynamic nature of the NVU.

Recent advances in biomaterials and microfluidic device fabrication allows for the recreation of a more physiologically relevant model. In this prospective, we primarily focus on the various approaches to create $3 \mathrm{D} \mathrm{BBB}$ tubular model designs (vessel formation, structural support, physical characteristics), then evaluate future directions and challenges. A word about terminology related to the use of the word microvessels. In its broadest definition, the sizes of blood vessels in the human body range from capillaries $(<8 \mu \mathrm{m})$, microvessels $(<1 \mathrm{~mm})$, small vessels (1-6 mm), and large vessels ( $>6 \mathrm{~mm}$ in diameter) (Chang and Niklason, 2017; Zhou et al., 2020). Cross-sectional measurements of brain microvessels derived from casting studies of human brains shows arterioles and arteries in the range of 50$240 \mu \mathrm{m}$ while post-capillary venules and veins range from 20 to 380 Mm (Duvernoy et al., 1981; Reina-De La Torre et al., 1998). A large majority of the vasculature in the brain is capillary which are found to range from 3 to $7.5 \mu \mathrm{m}$ (Duvernoy et al., 1981; Reina-De La Torre et al., 1998). Therefore, in this perspective the use of the term microvessel, refers to vascular structures that are sized below small vessels (under $1 \mathrm{~mm}$ ). We do not discuss in vitro organoids, another emerging area, due to the difficulty associated with applying fluid flow through microvessels at controlled and consistent flow rates. Overall, the next generation of $3 \mathrm{D}$ models has great promise to advance discoveries and treatments in areas of toxicology, drug delivery, neuropathology, infectious agents, and gene therapy.

\section{D BRAIN MICROVESSEL FABRICATION}

Multiple vascularization techniques have been developed to create $3 \mathrm{D}$ brain microvessel models in vitro, including $3 \mathrm{D}$ templating technique, 3D printing, and self-assembling-based techniques (Figure 1), which will be discussed below in detail.

\section{D Templating}

The most commonly used 3D microvessel modeling technique for the $\mathrm{BBB}$ is the $3 \mathrm{D}$ templating technique (Partyka et al., 2017; Grifno et al., 2019; Linville et al., 2019, 2020b,c; Bouhrira et al., 2020; Buzhdygan et al., 2020). This method creates a cylindrical microchannel surrounded by extracellular matrix (ECM), which can be lined with endothelial cells (ECs). The cylindrical microchannel is formed by inserting a cylindrical object (e.g., microneedle, thin rod, wire) into an ECM (hydrogel), typically collagen type I or fibrin, and removing the cylindrical object after the ECM is fully formed. An alternative methodology to accomplish a similar cylindrical microchannel is through Saffman-Taylor instability, in which a low viscosity liquid displaces a more viscous fluid from an ECM thus forming the cylindrical vessel (Herland et al., 2016). The 3D templating technique is only suitable to construct large linear blood vessels ranging from 60 to $700 \mu \mathrm{m}$ (Price et al., 2010; Bogorad et al., 2015; Wang et al., 2018). Then ECs are introduced into the inner channel to form a monolayer that lines the lumen. However, smaller vessel diameters increase the difficulties in sufficiently seeding EC to create a fully confluent vessel, though a recent study demonstrated the potential of using growth factor gradients to aid in endothelialization (Linville et al., 2016). Perhaps more crucially, this $3 \mathrm{D}$ templating technique is incapable of yielding the branched networks due to the manner in which the cylindrical object is removed from the ECM (Price et al., 2010; Buchanan et al., 2014). However, simplistic bifurcation models are possible such as that by Bouhrira et al. (2020). Although not a true branched vasculature, the sharp turn in fluid entry creates disturbed flow patterns in both steady and physiological waveforms (Bouhrira et al., 2020, 2021). Most 3D microvessel models consist of a single vessel, however, multiple side by side vessel scaffolds can also be created (Linville et al., 2020a). The dual vessel design can be used for access to the luminal vs. abluminal compartment. This configuration can allow for the generation of hydrostatic pressure and mechanical stress as well as to measure transendothelial-electrical resistance (TEER) (Partyka et al., 2017). 




FIGURE 1 | Techniques for fabrication of 3D brain microvessels and capillary-like structures. Techniques that have been used to create 3D BBB structures include 3D printing, 3D templating, and self-assembled microvascular networks. Advantages and disadvantages for each are presented as well as references for each technique used to model the BBB. $\left(^{\star}\right)$ Indicates models that use iPSC-derived ECs which are brain endothelial-like, as recent RNA-Sec data suggests these are more neuro-epithelial (Lu et al., 2021).

\section{D Printing}

In contrast to the templating technique, 3D printing employs principles of additive layer-by-layer deposition and sacrificial removal of filler material. Additionally, living cells can be printed in a 3D space using computer-aided design. There are two major technologies that have been used for the BBB: extrusion- and light-based bioprinting.

In the Extrusion-based printing (EBB) technique, biomaterials are extruded out of the printhead or nozzle by applying mechanical or pneumatic pressure. Inorganic substates are commonly extruded to create thin tubes which serve as a scaffold for a model of the BBB (Cucullo et al., 2007, 2011, 2013). Although some materials naturally create a porous structure during the extrusion process, most require additional micromachining to create uniform pores (Cucullo et al., 2011). Limitations include a thick wall which prevents the interactions of cells grown on the inner lumen and those on the outside surface and the size of extruded structures is limited by the nozzle size. For example, the dynamic in vitro blood-brain barrier (DIV-BBB) utilizes hollow polypropylene fibers which are 
$150 \mu \mathrm{m}$ thick and prevents any direct cell interactions (Cucullo et al., 2007, 2013). Many inorganic scaffolds are not translucent and thus visualizing the cells inside to verify a completely endothelialized structure is more challenging. Another aspect of inorganic substrates is that they are generally more rigid than organic ones. These inorganic scaffolds have not been used with a surrounding matrix but future models could incorporate a hydrogel to generate a parenchymal microenvironment. In addition to indirect printing of scaffolding templates, direct printing by extrusion is an alternative method which allows the incorporation of cells and biomolecules but has yet to be used in models of the BBB.

Light-based printing $(L B B)$ is a very fast and continuous $3 \mathrm{D}$ printing process which uses light as the energy source to crosslink biomaterials to form a scaffold. Most commonly utilized is UV or near UV wavelengths to polymerize hydrogels which may contain cells and proteins. This technique offers several advantages including very high resolution, creation of complex branching and tapering of vessel scaffolds in a 3D space (Grigoryan et al., 2019). With regards to BBB models, two-photon lithography has recently been employed to construct a series of tubes with an average diameter of 10 and $2 \mu \mathrm{m}$ wall thickness (Marino et al., 2018; Tricinci et al., 2020), close to capillary size. These structures resemble capillaries in size with the ability of a single endothelial cell to construct the lumen and for interactions with cells grown on the outer surface. As with the studies involving extruded tubes, these capillary tubes are not translucent and have not been used with a $3 \mathrm{D}$ matrix around the vessels. In addition to the fine scale resolution of two-photon lithography utilized by Marino et al. (2018) and Tricinci et al. (2020), LBB has promising capabilities for branched 3D BBB models. Using the Lumen $\mathrm{X}^{\mathrm{TM}}$, we demonstrate the ability to create scaffolds with a branching network. Furthermore, primary human brain microvascular endothelial cells completely line the scaffolding structure forming a lumen and the vessels transverse the matrix in the $Z$-axis (Figure 2).

\section{Self-Assembled Microvasculature}

Self-assembled-based techniques involve cells spontaneously organizing into capillary-like structures with the key presence of a lumen. These techniques can be split between angiogenic and vasculogenic approaches. Angiogenic strategies are based on cells invading into the surrounding hydrogel, whereby groups of ECs sprout, migrate, and organize to form new tubular configurations eventually forming blood vessels (Vailhé et al., 2001). The random sprouting through angiogenesis is more similar to in vivo biological processes. However, a limitation associated with angiogenesis is the replicability between constructs. Vessels sprout spontaneously, in different ways every time and some create overly dense networks (Haring and Johnson, 2020). The formation of these vessels is best with the structural support of fibroblasts or pericytes, which makes them a natural model for recreating the BBB (Campisi et al., 2018; Lee et al., 2020). Additionally, most angiogenic-based models have been used only with interstitial flow and higher capillary level shear stresses are difficult to achieve due to the angle of angiogenic spouts from the main vessels. Vasculogenic approaches involve culturing endothelial cells and pericytes within a hydrogel and allowing for the formation of microvessels over time. This strategy has been used to create capillary scale BBB-forming microvessels within collagen/hyaluronan composite hydrogels (Partyka et al., 2019; Tran et al., 2020). Similar to the angiogenic method, the vasculogenic protocol prohibits patterning of complex topologies since the structures are formed spontaneously. However, the application of interstitial flow can align these microvessels in a manner similar to non-BBB microvessels (Morin et al., 2014).

\section{BIOLOGICAL MATRICES TO SUPPORT PERFUSABLE MICROVESSELS}

The fabricating materials used to design the microvessel must be biocompatible. In $3 \mathrm{D}$ bioprinting, the bio-inks must retain the biological, physical and mechanical requirements during the operation of printing. Bio-inks with or without living cells are commonly used to configure scaffolds with microvessel structures. Generally, these crosslinkable substances include hydrogels such as collagen, gelatin, fibrin, alginate, chitosan, poly (ethylene glycol)-PEG hydrogels, poly(2-hydroxyethyl methacrylate)-pHEMA and poloxamers (Charnley et al., 2009; Wüst et al., 2011; Billiet et al., 2012; Vasile et al., 2020). These hydrogels have different properties for cell culture and growth. Some bio-inks are available in varying molecular weights which can impact the stiffness of the surrounding ECM. While stiffer substrates can be easier to manipulate, substrate stiffness and matrix density can affect EC spreading in some cases preventing full formation of endothelial cell-cell connections. Although, combining collagen hydrogels and polymer crosslinkers can improve cell attachment and the density of the collagen can encourage optimal endothelial sprouting (Crosby and Zoldan, 2019). It may also be necessary to include additional ECM components such as fibronectin, laminin, poly-lysine and large glycosaminoglycans (i.e., hyaluronic acid) to reinforce cell adhesion. Bio-inks are commonly mixed with living cells prior to the crosslinking process thus creating a scaffolding structure with cells embedded in the matrix.

\section{FLOW DYNAMICS AND STABILITY OF MICROVESSELS}

The cerebral vascular system exhibits different shear stress levels depending on the rate and velocity of blood flow. The typical physiological shear stress level of healthy arteries range from 10 to 70 dynes/cm (Saili et al., 2017), whereas healthy veins range from 1 to 6 dynes/cm² (Fung, 1997; Malek et al., 1999; Paszkowiak and Dardik, 2003). In brain capillaries, the blood flow is mostly heterogenous and have a broad range of shear stress levels due to the variation of vessel diameters (Mairey et al., 2006).

In particular, hemodynamic shear stress is known to modify morphology differentiation, reorganization, alignment and remodeling behaviors of EC that are significant for microvessel formation. Higher shear stress promotes EC survival and cause cells to align parallel with the direction of flow, while 


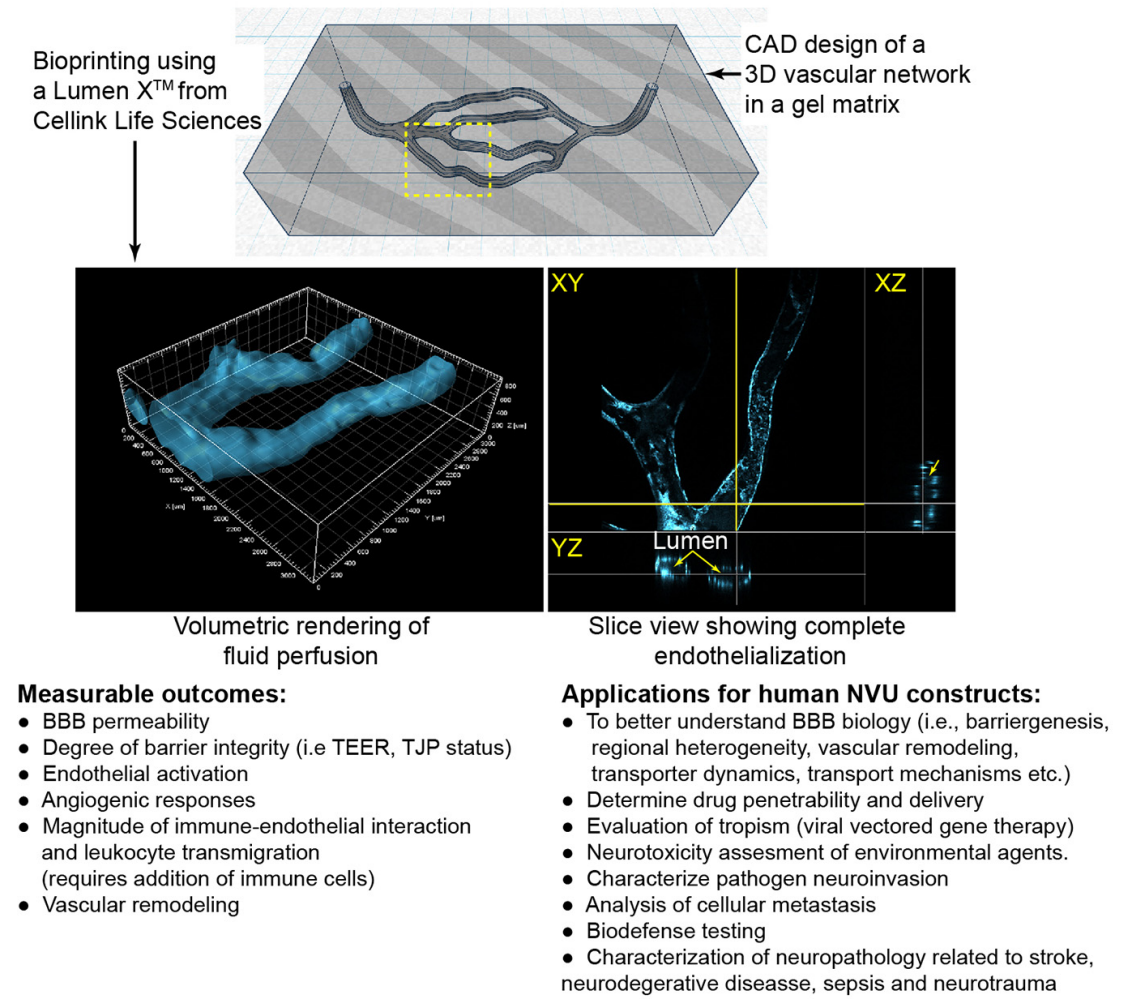

FIGURE 2 | Example of light-assistant bioprinting of 3D constructs and endothelialization with primary human brain microvascular endothelial cells (hBMVECs). 3D vascular scaffold was created using computer aided design (CAD) software and then were printed of PEGDA-GelMA using the LumenX'M . hBMVECs were grown under perfusion until completely confluent. Vessels ranged from 225 to $400 \mu \mathrm{m}$ in diameter. Possible applications and measurable outcomes for 3D constructs are described.

lower shear stress levels enhances EC apoptosis, proliferation, permeability, and shape change leading to vasoconstriction, coagulation, and platelet aggregation (Krizanac-Bengez et al., 2004). The shear stress, $\tau$, applied to ECs by laminar flow of culture medium, which is a Newtonian fluid, can be calculated using the Poiseuille equation (Lipowsky, 1995; Fung, 1997; Malek et al., 1999; Paszkowiak and Dardik, 2003; Galpayage Dona et al., 2020a).

$$
\tau=\frac{4 \mu Q}{\pi r^{3}}
$$

where $\mu$ is the viscosity of the blood, ( $\mu \sim 0.0035$ Pa.s), $Q$ is the blood flow rate and $r$ is the vessel radius.

Shear stress is a critical physiological parameter, however, perfusing $3 \mathrm{D}$ gels poses unique challenges relate to the mechanical properties (i.e., stiffness, stress relaxation, degradation, self-healing). It is important to note that few $3 \mathrm{D}$ brain microvessel models support physiological shear stresses (Cucullo et al., 2007, 2011, 2013). This may be in part due to the lack of matrices able to support the higher flow rates and the difficulties in obtaining a leak-free closed system. For that purpose, the future of 3D printed microvascular structure must address the necessity of higher shear stress level and mechanical properties of gel materials.
Studies on peripheral ECs have used different fluid flow patterns such as laminar, pulsatile, and turbulent flow to regulate shear stress of the $3 \mathrm{D}$ printed complex microstructure of a porous scaffold to employ well-formed EC monolayer (Song et al., 2005; Shao et al., 2009; Cui et al., 2019; Kinstlinger et al., 2020). These types of in vitro microfluidic studies are lacking in the field of the cerebral vasculature biology. However, Bouhrira et al. (2021) designed a flow system capable of generating complex, physiologically relevant flow profiles in a linear 3D BBB model. Branched networks and varying diameters adds a layer of intricacy to the shear stresses in vasculature structures. For instance, Kinstlinger et al. (2020) studied the flow rate of different dendritic vascular networks and observed a significant deviation between theoretical and experimental flow rate and shear stress values. These changes were explained by the fact of a small deviation of vascular radius through the network lead to a significant change of shear stress value (Eq. 1) (Kinstlinger et al., 2020). In regard to self-assembled networks, shear stress offered a tunable parameter for controlling bifurcations and branching. Perhaps appliable to brain ECs, Ueda et al. (2004) showed in Bovine pulmonary microvascular endothelial cells that the number of bifurcations and endpoints increased for networks exposed to shear stress, whereas the number of bifurcations alone increased for networks not exposed to shear stress. Moreover, the stability of the vessel can be achieved by addition of drainage 
channels or tapering the vessels (Khan and Sefton, 2011), by controlling the pressure of the vessel along the length of it.

Important to consider that in vivo, ECs experience the mechanical forces from the fluidic movement and interactions with blood components. Blood, in contrast to culture medium, is non-Newtonian and its boundary layer is affected by the thickness of the cell free layer, a phenomenon caused by red blood cell streaming (shear thinning). Multiple factors including hematocrit and vessel diameter, a relationship referred to as the Fahraeus-Lindquist effect, alter the magnitude and dynamics of shear stress applied to the endothelium. Additionally, the glycocalyx contributes to the homogenous blood flow distribution and mediating interactions between red blood cells with the microvasculature wall (Secomb et al., 1998; McClatchey et al., 2016). Therefore, incorporation of blood is necessary to gain a better understanding of how shear stress affects barrier structure and function.

\section{PERSPECTIVES, FUTURE DIRECTIONS, AND CONCLUSION}

Advances in tissue engineering and microfabrication enhance our ability to create new models of the BBB that more accurately mimic the in vivo structure and environment. Key areas of growth for the field include capillary structures capable of maintaining physiological shear stress levels, branched and multi-caliber vessel networks in a 3D space, as well as the inclusion of functional neurons in various anatomical configurations. To date, no 3D microfluidic model of the BBB incorporates functional neurons to create a true neurovascular unit.

In corporation of analytic measurements is an area for future improvement. For instance, microfluidic based electrical impedance techniques have been widely used to characterize the barrier related parameters and cellular and physical properties of cells (Cucullo et al., 2008; Douville et al., 2010; Szulcek et al., 2014; Reiss and Wegener, 2015; Bischoff et al., 2016; Galpayage Dona et al., 2017, 2020b), but few 3D models have realtime continuous measurements (Cucullo et al., 2007). Instead most rely on electrodes inserted into ports connected to the inner and outer luminal compartment requiring cessation of perfusion to obtained single timepoint measurements. New innovative designs that incorporate micro electrodes into these 3D spaces could yield outputs in real-time. Additional measurable parameters could include evaluations of metabolites, nitric oxide etc.

For the formation of new 3D scaffolding, one potential area of exploration includes the use of degradable materials (Qiu et al., 2019). This methodology has the potential to create a solid foundation for brain microvessels that afterward can be dissolved leaving the vessel behind. Also, 3D bioprinters have the capability to meet the demands of creating novel 3D branched and multi-caliber vessel networks. Although 3D bio-printers have high precision and reproducibility, the majority of printable networks have not reached capillary size yet. The developmental of new bioinks and devices with greater resolution can overcome existing hurdles.
Another primary challenge facing the field is the selection of an appropriate cell source to mimic brain endothelium. A recent study used RNA-sequencing demonstrated that iPS-derived cells used for BBB models are more epithelial in nature, which explains their ability to exhibit high TEER values ( $\mathrm{Lu}$ et al., 2021). Therefore, a better understanding of the differentiation programming of iPS-derived cells is needed to produce a brain endothelial phenotype. Another alternative to immortalized cell lines such as hCMEC/D3 is primary cells, though these sources are scarce. Primary adult human brain endothelial cells are in short supply commercially and healthy tissue derived from resections in surgical treatment of epilepsy are becoming less common (Bernas et al., 2010). Conversely, human brain endothelial cells isolated from fetal tissue have properties similar to adult primary cells and can be obtained from a wider range of donors and sexes (Andrews et al., 2018). An additional advantage from the use of human fetal tissue is the ability to isolate multiple cells of the neurovascular unit from the same donor (Andrews et al., 2018).

In summary, 3D tissue engineered models of the NVU requires the intersection of multiple fields of study, such as engineering, material science, cell biology, microfabrication and specialized expertise depending on application (toxicology, microbiology, neuroscience etc.). Undoubtedly, the advent of the next generation of physiologically akin NVU microfluidic models will greatly advance our ability to provide needed solutions for neurological diseases and disorders.

\section{DATA AVAILABILITY STATEMENT}

The raw data supporting the conclusions of this article will be made available by the authors, without undue reservation.

\section{AUTHOR CONTRIBUTIONS}

AMA, SR, and PG contributed to the conception, outline, and editing of this perspective. $\mathrm{BD}$ and $\mathrm{KT}$ edited the manuscript. TS and AA wrote sections of the manuscript. KG generated the construct shown in Figure 2 and wrote sections of the manuscript. $\mathrm{JH}$ performed the imaging and image analysis shown. All authors contributed to manuscript revision, read, and approved the submitted version.

\section{FUNDING}

This work was supported by the K01DA046308 (AMA), 5P30DA013429 (AMA), 5R01DA046833 (SR), and R01DA052970 (SR), and the Shriners Hospitals for Children 85180-PHI-20 (SR and AMA).

\section{ACKNOWLEDGMENTS}

We would like to acknowledge Hannah McGary for her contribution to the schematic in Figure 1. 


\section{REFERENCES}

Abbott, N. J. (2013). Blood-brain barrier structure and function and the challenges for cns drug delivery. J. Inherit Metab. Dis. 36, 437-449. doi: 10.1007/s10545013-9608-0

Abbott, N. J., Patabendige, A. A., Dolman, D. E., Yusof, S. R., and Begley, D. J. (2010). Structure and function of the blood-brain barrier. Neurobiol Dis. 37, 13-25. doi: 10.1016/j.nbd.2009.07.030

Abbott, N. J., Ronnback, L., and Hansson, E. (2006). Astrocyte-endothelial interactions at the blood-brain barrier. Nat. Rev. Neurosci. 7, 41-53. doi: 10. $1038 / \mathrm{nrn} 1824$

Aday, S., Cecchelli, R., Hallier-Vanuxeem, D., Dehouck, M. P., and Ferreira, L. (2016). Stem cell-based human blood-brain barrier models for drug discovery and delivery. Trends Biotechnol. 4, 382-393. doi: 10.1016/j.tibtech.2016.01.001

Andrews, A. M., Lutton, E. M., Cannella, L. A., Reichenbach, N., Razmpour, R., Seasock, M. J., et al. (2018). Characterization of human fetal brain endothelial cells reveals barrier properties suitable for in vitro modeling of the bbb with syngenic co-cultures. J. Cereb. Blood Flow Metab. 38, 888-903. doi: 10.1177/ $0271678 X 17708690$

Bazzoni, G., and Dejana, E. (2004). Endothelial cell-to-cell junctions: molecular organization and role in vascular homeostasis. Physiol. Rev. 84, 869-901. doi: 10.1152/physrev.00035.2003

Bernas, M. J., Cardoso, F. L., Daley, S. K., Weinand, M. E., Campos, A. R., Ferreira, A. J., et al. (2010). Establishment of primary cultures of human brain microvascular endothelial cells to provide an in vitro cellular model of the blood-brain barrier. Nat. Protoc. 5, 1265-1272. doi: 10.1038/nprot.2010.76

Bickel, U., Yoshikawa, T., and Pardridge, W. M. (2001). Delivery of peptides and proteins through the blood-brain barrier. Adv. Drug Delivery Rev. 46, 247-279.

Billiet, T., Vandenhaute, M., Schelfhout, J., Van Vlierberghe, S., and Dubruel, P. (2012). A review of trends and limitations in hydrogel-rapid prototyping for tissue engineering. Biomaterials 33, 6020-6041.

Bischoff, I., Hornburger, M. C., Mayer, B. A., Beyerle, A., Wegener, J., and Fürst, R. (2016). Pitfalls in assessing microvascular endothelial barrier function: impedance-based devices versus the classic macromolecular tracer assay. Sci. Rep. 6:23671.

Bogorad, M. I., DeStefano, J., Karlsson, J., Wong, A. D., Gerecht, S., and Searson, P. C. (2015). In vitro microvessel models. Lab Chip 15, 4242-4255.

Bonkowski, D., Katyshev, V., Balabanov, R. D., Borisov, A., and Dore-Duffy, P. (2011). The cns microvascular pericyte: Pericyte-astrocyte crosstalk in the regulation of tissue survival. Fluids Barriers CNS 8:8. doi: 10.1186/2045-81188-8

Bouhrira, N., DeOre, B. J., and Galie, P. A. (2021). Implementation and characterization of a physiologically relevant flow waveform in a $3 \mathrm{~d}$ microfluidic model of the blood-brain barrier. Biotechnol. Bioeng. 118, 2411-2421. doi: 10. 1002/bit.27719

Bouhrira, N., DeOre, B. J., Sazer, D. W., Chiaradia, Z., Miller, J. S., and Galie, P. A. (2020). Disturbed flow disrupts the blood-brain barrier in a $3 \mathrm{~d}$ bifurcation model. Biofabrication 12:025020. doi: 10.1088/1758-5090/ab5898

Buchanan, C. F., Voigt, E. E., Szot, C. S., Freeman, J. W., Vlachos, P. P., and Rylander, M. N. (2014). Three-dimensional microfluidic collagen hydrogels for investigating flow-mediated tumor-endothelial signaling and vascular organization. Tissue Eng. Part C Methods 20, 64-75.

Buzhdygan, T. P., DeOre, B. J., Baldwin-Leclair, A., Bullock, T. A., McGary, H. M., Khan, J. A., et al. (2020). The sars-cov-2 spike protein alters barrier function in $2 \mathrm{~d}$ static and $3 \mathrm{~d}$ microfluidic in-vitro models of the human blood-brain barrier. Neurobiol. Dis. 146, 105131. doi: 10.1016/j.nbd.2020.105131

Campisi, M., Shin, Y., Osaki, T., Hajal, C., Chiono, V., and Kamm, R. D. (2018). $3 \mathrm{~d}$ self-organized microvascular model of the human blood-brain barrier with endothelial cells, pericytes and astrocytes. Biomaterials 180, 117-129. doi: 10 . 1016/j.biomaterials.2018.07.014

Castro Dias, M., Mapunda, J. A., Vladymyrov, M., and Engelhardt, B. (2019). Structure and junctional complexes of endothelial, epithelial and glial brain barriers. Int. J. Mol. Sci. 20:5372. doi: 10.3390/ijms20215372

Chang, W. G., and Niklason, L. E. (2017). A short discourse on vascular tissue engineering. NPJ Regen Med. 2:7. doi: 1038/s41536-017-0011-6

Charnley, M., Textor, M., Khademhosseini, A., and Lutolf, M. P. (2009). Integration column: microwell arrays for mammalian cell culture. Integr. Biol. 1, 625-634.

Colgan, O. C., Ferguson, G., Collins, N. T., Murphy, R. P., Meade, G., Cahill, P. A., et al. (2007). Regulation of bovine brain microvascular endothelial tight junction assembly and barrier function by laminar shear stress. Am. J. Physiol. Heart. Circ Physiol 292, H3190-H3197. doi: 10.1152/ajpheart.01177.2006

Crosby, C. O., and Zoldan, J. (2019). Mimicking the physical cues of the ecm in angiogenic biomaterials. Regen Biomater. 6, 61-73. doi: 10.1093/rb/rbz003

Cucullo, L., Couraud, P.-O., Weksler, B., Romero, I.-A., Hossain, M., Rapp, E., et al. (2008). Immortalized human brain endothelial cells and flow-based vascular modeling: a marriage of convenience for rational neurovascular studies. J. Cereb. Blood Flow Metab. 28, 312-328.

Cucullo, L., Hossain, M., Rapp, E., Manders, T., Marchi, N., and Janigro, D. (2007). Development of a humanized in vitro blood-brain barrier model to screen for brain penetration of antiepileptic drugs. Epilepsia 48, 505-516. doi: 10.1111/j. 1528-1167.2006.00960.x

Cucullo, L., Hossain, M., Tierney, W., and Janigro, D. (2013). A new dynamic in vitro modular capillaries-venules modular system: Cerebrovascular physiology in a box. BMC Neurosci. 14:18. doi: 10.1186/1471-2202-14-18

Cucullo, L., Marchi, N., Hossain, M., and Janigro, D. (2011). A dynamic in vitro bbb model for the study of immune cell trafficking into the central nervous system. J. Cereb. Blood Flow Metab. 31, 767-777. doi: 10.1038/jcbfm.2010.162

Cui, H., Zhu, W., Huang, Y., Liu, C., Yu, Z.-X., Nowicki, M., et al. (2019). In vitro and in vivo evaluation of $3 \mathrm{~d}$ bioprinted small-diameter vasculature with smooth muscle and endothelium. Biofabrication 12:015004.

Douville, N. J., Tung, Y.-C., Li, R., Wang, J. D., El-Sayed, M. E., and Takayama, S. (2010). Fabrication of two-layered channel system with embedded electrodes to measure resistance across epithelial and endothelial barriers. Anal. Chem. 82, 2505-2511.

Duvernoy, H. M., Delon, S., and Vannson, J. L. (1981). Cortical blood vessels of the human brain. Brain Res. Bull. 7, 519-579. doi: 10.1016/0361-9230(81)90007-1

Fung, Y. C. (1997). "Blood flow in arteries," in Biomechanics, (New York, NY: Springer), 108-205. doi: 10.1007/978-1-4757-2696-1_3

Galpayage Dona, KNU., Du, E., Carlsson, L. A., Fletcher, D. M., and Boardman, R. P. (2020a). Modeling of water wicking along fiber/matrix interface voids in unidirectional carbon/vinyl ester composites. Microfluid. Nanofluid. 24:31.

Galpayage Dona, KNU., Du, E., and Wei, J. (2020b). An impedimetric assay for the identification of abnormal mitochondrial dynamics in living cells. Electrophoresis 42, 163-170.

Galpayage Dona, KNU., Liu, J., Qiang, Y., Du, E., and Lau, A. W. C. (2017). "Electrical equivalent circuit model of sickle cell," in Proceedings of the ASME 2017 International Mechanical Engineering Congress and Exposition; 2017. (santa barbara, CA: American Society of Mechanical Engineers Digital Collection).

Grifno, G. N., Farrell, A. M., Linville, R. M., Arevalo, D., Kim, J. H., Gu, L., et al. (2019). Tissue-engineered blood-brain barrier models via directed differentiation of human induced pluripotent stem cells. Sci. Rep. 9:13957. doi: 10.1038/s41598-019-50193-1

Grigoryan, B., Paulsen, S. J., Corbett, D. C., Sazer, D. W., Fortin, C. L., Zaita, A. J., et al. (2019). Multivascular networks and functional intravascular topologies within biocompatible hydrogels. Science 364, 458-464. doi: 10.1126/science. aav9750

Gundersen, G. A., Vindedal, G. F., Skare, O., and Nagelhus, E. A. (2014). Evidence that pericytes regulate aquaporin-4 polarization in mouse cortical astrocytes. Brain Struct. Funct. 219, 2181-2186. doi: 10.1007/s00429-013-0629-0

Haring, A. P., and Johnson, B. N. (2020). "Brain-on-a-chip systems for modeling disease pathogenesis," in Organ-on-a-chip, ed. F. Geraghty (Cambridge, MA: Academic Press), 215-232.

Herland, A., van der Meer, A. D., FitzGerald, E. A., Park, T. E., Sleeboom, J. J., and Ingber, D. E. (2016). Distinct contributions of astrocytes and pericytes to neuroinflammation identified in a $3 \mathrm{~d}$ human blood-brain barrier on a chip. PLoS One 11:e0150360. doi: 10.1371/journal.pone.0150360

Khan, O. F., and Sefton, M. V. (2011). Endothelial cell behaviour within a microfluidic mimic of the flow channels of a modular tissue engineered construct. Biomed. Microdevices 13, 69-87.

Kinstlinger, I. S., Saxton, S. H., Calderon, G. A., Ruiz, K. V., Yalacki, D. R., Deme, P. R., et al. (2020). Generation of model tissues with dendritic vascular networks via sacrificial laser-sintered carbohydrate templates. Nat. Biomed. Eng. 4, 916-932.

Krizanac-Bengez, L., Mayberg, M. R., and Janigro, D. (2004). The cerebral vasculature as a therapeutic target for neurological disorders and the role of shear stress in vascular homeostatis and pathophysiology. Neurol Res. 26, 846-853. doi: 10.1179/016164104X3789 
Lee, S., Chung, M., Lee, S. R., and Jeon, N. L. (2020). 3d brain angiogenesis model to reconstitute functional human blood-brain barrier in vitro. Biotechnol. Bioeng. 117, 748-762. doi: 10.1002/bit.27224

Linville, R. M., Arevalo, D., Maressa, J. C., Zhao, N., and Searson, P. C. (2020a). Three-dimensional induced pluripotent stem-cell models of human brain angiogenesis. Microvasc. Res. 132:104042. doi: 10.1016/j.mvr.2020.104042

Linville, R. M., Boland, N. F., Covarrubias, G., Price, G. M., and Tien, J. (2016). Physical and chemical signals that promote vascularization of capillary-scale channels. Cell Mol. Bioeng. 9, 73-84. doi: 10.1007/s12195-016-0429-8

Linville, R. M., DeStefano, J. G., Nerenberg, R. F., Grifno, G. N., Ye, R., Gallagher, E., et al. (2020b). Long-term cryopreservation preserves blood-brain barrier phenotype of ipsc-derived brain microvascular endothelial cells and three-dimensional microvessels. Mol. Pharm. 17, 3425-3434. doi: 10.1021/acs. molpharmaceut.0c00484

Linville, R. M., DeStefano, J. G., Sklar, M. B., Chu, C., Walczak, P., and Searson, P. C. (2020c). Modeling hyperosmotic blood-brain barrier opening within human tissue-engineered in vitro brain microvessels. J. Cereb. Blood Flow Metab. 40, 1517-1532. doi: 10.1177/0271678X19867980

Linville, R. M., DeStefano, J. G., Sklar, M. B., Xu, Z., Farrell, A. M., Bogorad, M. I., et al. (2019). Human ipsc-derived blood-brain barrier microvessels: validation of barrier function and endothelial cell behavior. Biomaterials 190-191, 24-37. doi: 10.1016/j.biomaterials.2018.10.023

Lipowsky, H. H. (1995). "Shear stress in the circulation," in Flow-Dependent Regulation of Vascular Function, eds J. A. Bevan, G. Kaley, and G. M. Rubanyi (New York, NY: Springer), 28-45.

Lochhead, J. J., Yang, J., Ronaldson, P. T., and Davis, T. P. (2020). Structure, function, and regulation of the blood-brain barrier tight junction in central nervous system disorders. Front. Physiol. 11:914. doi: 10.3389/fphys.2020.00914

Lu, T. M., Houghton, S., Magdeldin, T., Duran, J. G. B., Minotti, A. P., Snead, A., et al. (2021). Pluripotent stem cell-derived epithelium misidentified as brain microvascular endothelium requires ets factors to acquire vascular fate. Proc. Natl. Acad. Sci. U.S.A. 11:914. doi: 10.1073/pnas.2016950118

Mairey, E., Genovesio, A., Donnadieu, E., Bernard, C., Jaubert, F., Pinard, E., et al. (2006). Cerebral microcirculation shear stress levels determine neisseria meningitidis attachment sites along the blood-brain barrier. J. Exp. Med. 203, 1939-1950. doi: 10.1084/jem.20060482

Malek, A. M., Alper, S. L., and Izumo, S. (1999). Hemodynamic shear stress and its role in atherosclerosis. JAMA 282, 2035-2042.

Marino, A., Tricinci, O., Battaglini, M., Filippeschi, C., Mattoli, V., Sinibaldi, E., et al. (2018). A 3d real-scale, biomimetic, and biohybrid model of the blood-brain barrier fabricated through two-photon lithography. Small 14:10.1002/smll.201702959. doi: 10.1002/smll.201702959

McClatchey, P. M., Schafer, M., Hunter, K. S., and Reusch, J. E. (2016). The endothelial glycocalyx promotes homogenous blood flow distribution within the microvasculature. Am. J. Physiol. Heart Circ. Physiol. 311, H168-H176. doi: 10.1152/ajpheart.00132.2016

Morin, K. T., Dries-Devlin, J. L., and Tranquillo, R. T. (2014). Engineered microvessels with strong alignment and high lumen density via cell-induced fibrin gel compaction and interstitial flow. Tissue Eng. Part A. 20, 553-565. doi: 10.1089/ten.TEA.2013.0262

Partyka, P. P., Godsey, G. A., Galie, J. R., Kosciuk, M. C., Acharya, N. K., Nagele, R. G., et al. (2017). Mechanical stress regulates transport in a compliant $3 \mathrm{~d}$ model of the blood-brain barrier. Biomaterials 115, 30-39. doi: 10.1016/j. biomaterials.2016.11.012

Partyka, P. P., Jin, Y., Bouyer, J., DaSilva, A., Godsey, G. A., Nagele, R. G., et al. (2019). Harnessing neurovascular interaction to guide axon growth. Sci. Rep. 9:2190. doi: 10.1038/s41598-019-38558-y

Paszkowiak, J. J., and Dardik, A. (2003). Arterial wall shear stress: Observations from the bench to the bedside. Vasc. Endovas. Surg. 37, 47-57.

Price, G. M., Wong, K. H., Truslow, J. G., Leung, A. D., Acharya, C., and Tien, J. (2010). Effect of mechanical factors on the function of engineered human blood microvessels in microfluidic collagen gels. Biomaterials 31, 6182-6189.

Qiu, Y. L., Chen, X., Hou, Y. L., Hou, Y. J., Tian, S. B., Chen, Y. H., et al. (2019). Characterization of different biodegradable scaffolds in tissue engineering. Mol. Med. Rep. 19, 4043-4056. doi: 10.3892/mmr.2019.10066

Reina-De La Torre, F., Rodriguez-Baeza, A., and Sahuquillo-Barris, J. (1998). Morphological characteristics and distribution pattern of the arterial vessels in human cerebral cortex: a scanning electron microscope study. Anat. Rec.
251, 87-96. doi: 10.1002/(SICI)1097-0185(199805)251:1<87::AID-AR14<3.0 $\mathrm{CO} ; 2-7$

Reiss, B and Wegener, J. (2015). Impedance analysis of different cell monolayers grown on gold-film electrodes. Annu. Int. Conf. IEEE Eng. Med. Biol. Soc. 2015, 7079-7082.

Saili, K. S., Zurlinden, T. J., Schwab, A. J., Silvin, A., Baker, N. C., Hunter, E. S. III, et al. (2017). Blood-brain barrier development: systems modeling and predictive toxicology. Birth Defects Res. 109, 1680-1710.

Secomb, T. W., Hsu, R., and Pries, A. R. (1998). A model for red blood cell motion in glycocalyx-lined capillaries. Am. J. Physiol. 274, H1016-H1022. doi: 10.1152/ajpheart.1998.274.3.H1016

Shao, J., Wu, L., Wu, J., Zheng, Y., Zhao, H., Jin, Q., et al. (2009). Integrated microfluidic chip for endothelial cells culture and analysis exposed to a pulsatile and oscillatory shear stress. Lab on a Chip 9, 3118-3125.

Song, H. W., Foreman, K. L., Gastfriend, B. D., Kuo, J. S., Palecek, S. P., and Shusta, E. V. (2020). Transcriptomic comparison of human and mouse brain microvessels. Sci. Rep. 10:12358. doi: 10.1038/s41598-020-69096-7

Song, J. W., Gu, W., Futai, N., Warner, K. A., Nor, J. E., and Takayama, S. (2005). Computer-controlled microcirculatory support system for endothelial cell culture and shearing. Anal. Chem. 77, 3993-3999.

Szulcek, R., Bogaard, H. J., and van Nieuw Amerongen, G. P. (2014). Electric cellsubstrate impedance sensing for the quantification of endothelial proliferation, barrier function, and motility. J. Vis. Exp. JoVE 28:51300.

Tran, K. A., Partyka, P. P., Jin, Y., Bouyer, J., Fischer, I., and Galie, P. A. (2020). Vascularization of self-assembled peptide scaffolds for spinal cord injury repair. Acta Biomater. 104, 76-84. doi: 10.1016/j.actbio.2019.12.033

Tricinci, O., De Pasquale, D., Marino, A., Battaglini, M., Pucci, C., and Ciofani, G. (2020). A 3d biohybrid real-scale model of the brain cancer microenvironment for advanced in vitro testing. Adv. Mater. Technol. 5:2000540. doi: 10.1002/ admt.202000540

Ueda, A., Koga, M., Ikeda, M., Kudo, S., and Tanishita, K. (2004). Effect of shear stress on microvessel network formation of endothelial cells with in vitro three-dimensional model. Am. J. Physiol. Heart Circ. Physiol. 287, H994-H1002.

Vailhé, B., Vittet, D., and Feige, J.-J. (2001). In vitro models of vasculogenesis and angiogenesis. Lab. Invest. 81, 439-452.

Vasile, C., Pamfil, D., Stoleru, E., and Baican, M. (2020). New developments in medical applications of hybrid hydrogels containing natural polymers. Molecules 25:1539.

Wang, X., Sun, Q., and Pei, J. (2018). Microfluidic-based 3d engineered microvascular networks and their applications in vascularized microtumor models. Micromachines 9:493

Wüst, S., Müller, R., and Hofmann, S. (2011). Controlled positioning of cells in biomaterials-approaches towards $3 \mathrm{~d}$ tissue printing. J. Funct. Biomater. 2, 119-154.

Zhang, J. H., Badaut, J., Tang, J., Obenaus, A., Hartman, R., and Pearce, W. J. (2012). The vascular neural network-a new paradigm in stroke pathophysiology. Nat. Rev. Neurol. 8, 711-716. doi: 10.1038/nrneurol.2012.210

Zhou, X., Nowicki, M., Sun, H., Hann, S. Y., Cui, H., Esworthy, T., et al. (2020). 3d bioprinting-tunable small-diameter blood vessels with biomimetic biphasic cell layers. ACS Appl. Mater. Interf. 12, 45904-45915. doi: 10.1021/acsami.0c14871

Conflict of Interest: The authors declare that the research was conducted in the absence of any commercial or financial relationships that could be construed as a potential conflict of interest.

Publisher's Note: All claims expressed in this article are solely those of the authors and do not necessarily represent those of their affiliated organizations, or those of the publisher, the editors and the reviewers. Any product that may be evaluated in this article, or claim that may be made by its manufacturer, is not guaranteed or endorsed by the publisher.

Copyright (C) 2021 Galpayage Dona, Hale, Salako, Anandanatarajan, Tran, DeOre, Galie, Ramirez and Andrews. This is an open-access article distributed under the terms of the Creative Commons Attribution License (CC BY). The use, distribution or reproduction in other forums is permitted, provided the original author(s) and the copyright owner(s) are credited and that the original publication in this journal is cited, in accordance with accepted academic practice. No use, distribution or reproduction is permitted which does not comply with these terms. 